\title{
Exploiting immune-dependent effects of microtubule-targeting agents to improve efficacy and tolerability of cancer treatment
}

\author{
Angela Flavia Serpico ${ }^{1,2}$, Roberta Visconti ${ }^{3}$ and Domenico Grieco ${ }^{1,4}$
}

\begin{abstract}
Microtubule-targeting agents (MTAs), like taxanes and vinca alkaloids, are tubulin-binding drugs that are very effective in the treatment of various types of cancers. In cell cultures, these drugs appear to affect assembly of the mitotic spindle and to delay progression through mitosis and this correlates with their ability to induce cell death. Their clinical efficacy is, however, limited by resistance and toxicity. For these reasons, other spindle-targeting drugs, affecting proteins such as certain kinesins like Eg5 and CENP-E, or kinases like PIk1, Aurora A and B, have been developed as an alternative to MTAs. However, these attempts have disappointed in the clinic since these drugs show poor anticancer activity and toxicity ahead of positive effects. In addition, whether efficacy of MTAs in cancer treatment is solely due to their ability to delay mitosis progression remains controversial. Here we discuss recent findings indicating that the taxane paclitaxel can promote a proinflammatory response by activation of innate immunity. We further describe how this can help adaptive antitumor immune response and suggest, on this basis and on the recent success of immune checkpoint inhibitors in cancer treatment, that a combination therapy based on low doses of taxanes and immune checkpoint inhibitors may be of high clinical advantage in terms of wide applicability, reduced toxicity, and increased antitumor response.
\end{abstract}

\section{Introduction}

MTAs have been introduced in cancer therapy since several years and are still among the most widely used antitumor drugs, utilized alone or in combination with other antiblastic drugs, to treat different cancers $^{1-3}$. In addition, MTAs are still an essential resource as second line treatments and for the treatment of tumors that lack known specific molecular targets and cannot benefit from recent advances in targeted therapy. Indeed, the taxane docetaxel has been approved for treatment of castration resistant prostate cancer and of triple-negative breast cancer, alone or in combination with other drugs ${ }^{4}$.

MTAs bind $\beta$-tubulin and severely affect microtubule dynamics through different mechanisms. Taxanes

\footnotetext{
Correspondence: Domenico Grieco (domenico.grieco@unina.it)

${ }^{1}$ CEINGE Biotecnologie Avanzate, Naples, Italy

2DMMBM, University of Naples "Federico II", Naples, Italy

Full list of author information is available at the end of the article

Edited by $\mathrm{G}$. Melino
}

stabilize microtubules while vinca alkaloids hamper microtubule polymerization. Thus, MTAs interfere with many key cellular processes. In interphase, the intracellular transport of proteins, vesicles, and organelles along trucks formed by microtubule fibers are deeply affected by $\mathrm{MTAs}^{5-7}$. In mitosis, the microtubular cytoskeleton is profoundly rearranged to form the mitotic spindle, the structure required to segregate replicated chromosomes during cell division, and this is also deeply affected by MTAs $^{8}$. By altering normal mitotic spindle assembly, MTAs activate the spindle assembly checkpoint (SAC), a safeguard mechanism that prevents errors in chromosome segregation and generation of aneuploid cells by delaying mitosis exit when spindle assembly is impaired (Fig. 1; high taxanes) ${ }^{9,10}$. When spindle assembly is incomplete or impaired, SAC effector proteins, like BubR1 and Mad2, bind Cdc20, a coactivator of the ubiquitin ligase Anaphase-Promoting Complex/Cyclosome (APC/ $\mathrm{C})$, to form a mitotic checkpoint complex (MCC). MCC

\section{(c) The Author(s) 2020}

(c) (i) Open Access This article is licensed under a Creative Commons Attribution 4.0 International License, which permits use, sharing, adaptation, distribution and reproduction cc) in any medium or format, as long as you give appropriate credit to the original author(s) and the source, provide a link to the Creative Commons license, and indicate if changes were made. The images or other third party material in this article are included in the article's Creative Commons license, unless indicated otherwise in a credit line to the material. If material is not included in the article's Creative Commons license and your intended use is not permitted by statutory regulation or exceeds the permitted use, you will need to obtain permission directly from the copyright holder. To view a copy of this license, visit http://creativecommons.org/licenses/by/4.0/. 


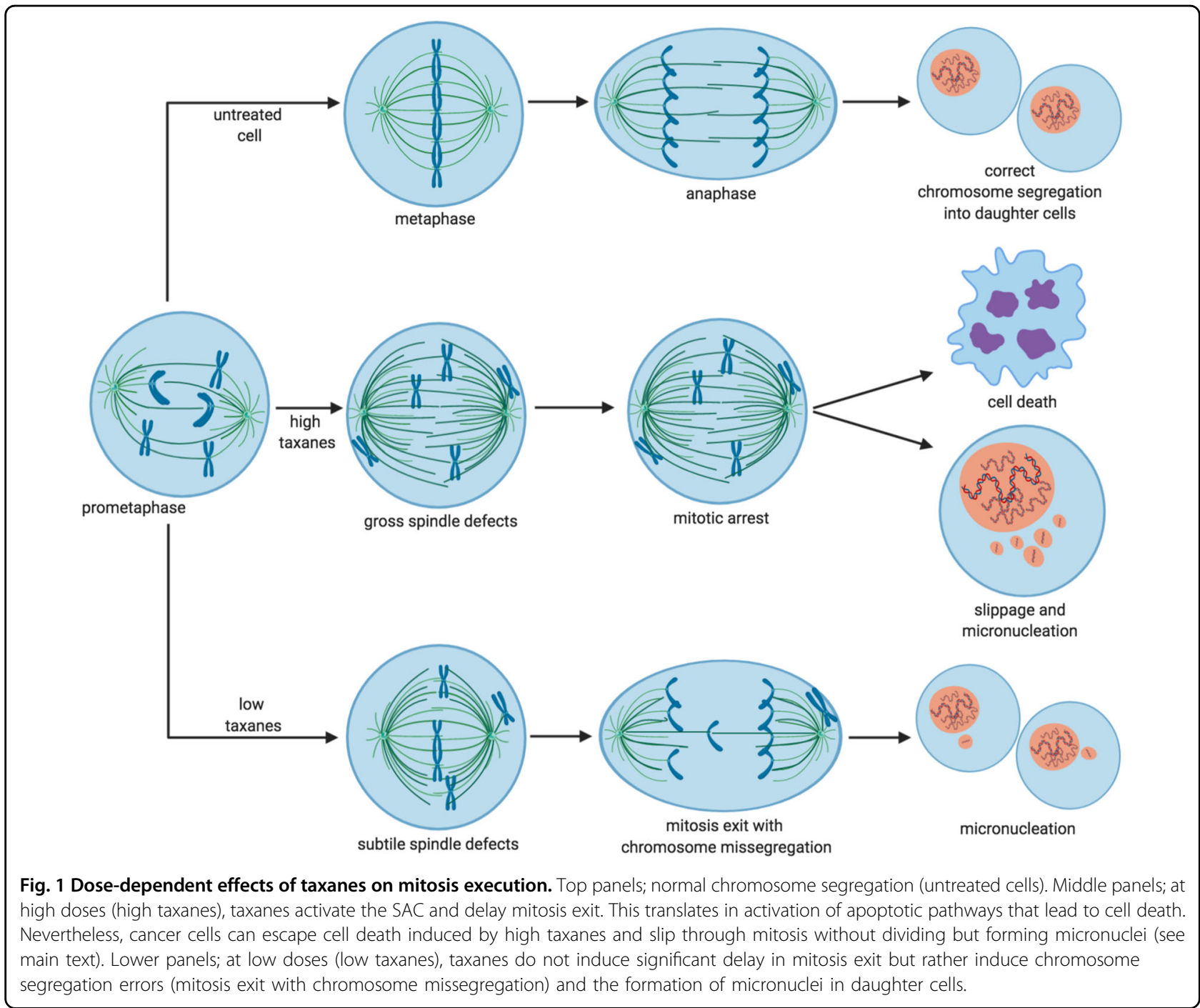

inhibits $\mathrm{APC} / \mathrm{C}$-dependent degradation and inactivation of the master mitotic kinase cyclin B-dependent kinase (Cdk) 1 and of the anaphase inhibitor securin, arresting cells in mitosis. However, after prolonged SAC-dependent delay in mitosis, cells activate cell-death programs ${ }^{10-14}$. Indeed, in cell cultures, MTA-dependent mitotic perturbance and delayed progression through mitosis correlate with the MTA ability of inducing cell death, thus providing a mechanistic rationale for the therapeutic effects of these drugs. However, as discussed later, mitotic delay may not be the only mechanism by which MTAs kill cancer cells.

\section{Limitations of MTA-based cancer therapy}

In the clinic, most patients immediately respond to treatment with MTAs, with very few cases of naive resistance against these drugs ${ }^{15}$. Unfortunately, patients that initially respond well to MTA therapy may later develop resistance to treatment. The mechanisms of acquired resistance to MTAs are several, spanning from the more general upregulation of the $\mathrm{ABC}$ transmembrane efflux transporters to the much more drug specific, "on-target" mutations of microtubule forming or binding proteins. For example, the response to MTAs can be limited by mutations or by altered expression of the microtubule building blocks $\alpha$ - and $\beta$-tubulin ${ }^{4,15,16}$. A more common way of resistance to MTAs, however, is believed to derive from an adaptation mechanism known as "mitotic slippage"17. Indeed, even in the presence of therapeutic concentrations of MTAs, cells can override SAC-induced mitotic arrest and slip out of mitosis (Fig. 1; high taxanes). This is mainly due to a progressive loss of Cdk1 activity, during arrest, to a point in which the SAC, that requires $\mathrm{Cdk} 1$ activity, cannot be held active anymore in preventing cyclin $\mathrm{B}$ degradation and mitosis exit ${ }^{18,19}$. MTA-treated cells can survive if they slip through mitosis before the threshold necessary to induce mitotic delaydependent cell death has been reached and either die 
afterwards, or stop proliferating, or give rise to more aggressive clones ${ }^{8,14}$. It can, therefore, be inferred that preventing mitotic slippage for a time sufficient enough to induce mitotic cell-death pathways could avoid this mechanism of resistance. To this end, it has been suggested that inhibiting $\mathrm{APC} / \mathrm{C}^{\mathrm{Cdc} 20}$, the ubiquitin ligase responsible for cyclin $\mathrm{B}$ and securin degradation and, therefore, crucial for mitosis exit, might help cancer cell killing in aid to MTA treatments ${ }^{18}$. However, the two so far described APC/C $\mathrm{C}^{\mathrm{Cdc} 20}$ inhibitors, TAME and Apcin, though promisingly efficient in blocking mitosis exit in experimental settings, are not yet available for clinical use and very recent observations cast doubts on their mechanism of action ${ }^{20-22}$. As an alternative to sustain MTA-induced mitotic arrest, we have proposed to combine MTAs with the Wee1 kinase inhibitor AZD-1775, based on a novel role we unveiled for Wee1 in regulating mitosis exit ${ }^{23,24}$. Of note, we proved that Wee1 genetic depletion substantially delayed mitotic exit and that chemically inhibiting Wee1 with AZD-1775 synergized with MTAs by further prolonging mitosis and increasing cell death in cancer cell lines and primary human lymphoblastic leukemia cells ${ }^{18,23}$.

Besides resistance, the use of MTAs can be substantially limited also by side effects, in some cases so severe to force to dose reduction or discontinuation. Above all, MTAs cause neutropenia and lymphopenia, a consequence of toxicity on cycling hematopoietic precursor cells, and neurotoxicity, likely by disruption of microtubule-mediated axonal transport in neurons ${ }^{25}$. To control this, new MTA formulations have been developed and tested to reduce doses, optimizing delivery, and distribution. The nanoparticle albumin-bound (nab)-paclitaxel is a solvent-free, colloidal suspension of the taxane paclitaxel, and human serum albumin, already approved for cancer therapy ${ }^{26}$. Microspheres and liposomes are currently tested as MTA vehicles ${ }^{27}$.

Looking for an alternative way to delay mitosis exit and promote cancer cell death, many spindle-targeting drugs, not directly targeting tubulin but rather microtubuleassociated proteins or kinases required for mitotic spindle assembly, have been developed ${ }^{28}$. Among them, several inhibitors of the kinesin superfamily proteins (KIFs) have been exploited. Kinesins and kinesin-related proteins make up a large superfamily of molecular motors responsible for the major microtubule- and ATPdependent transport pathways and some of them are particularly relevant for spindle assembly ${ }^{29,30}$. Disappointingly, however, most of mitotic KIF inhibitors tested, although able to perturb mitotic spindle assembly, do not kill cells efficiently ${ }^{29,30}$. A large number of molecules has also been developed to inhibit kinases like Plk1, Aurora A and Aurora B that are required for spindle assembly $^{31}$. Nevertheless, initial clinical trials with most of the Plk and Aurora inhibitors have not confirmed the promising preclinical efficacy and only very few drugs, such as the Aurora A kinase inhibitor alisertib, reached phase III trials for a wide variety of tumors, upon encouraging response rates in phase II trial. However, the positive results have been poorly confirmed in further trials $^{32,33}$.

\section{Immunotherapies targeting immune checkpoints}

Cancers are often infiltrated by a heterogeneous population of tumor-infiltrating immune cells and whether this produces pro- or antitumor effects is still matter of extensive investigation. IFN- $\gamma$-producing $\mathrm{CD} 4^{+} \mathrm{T}$ helper (Th) 1 cells, $\mathrm{CD}^{+} \mathrm{T}$ cells, natural killer (NK) cells, type 1 NK T cells, mature dendritic cells (DCs), and M1 macrophages could generate an antitumor response. On the other hand, $\mathrm{CD} 4^{+} \mathrm{Th} 2$ cells, $\mathrm{CD} 4^{+} \mathrm{T}$ regulatory (TReg) cells, type $2 \mathrm{NK} T$ cells, myeloid-derived suppressor cells, immature DCs, and M2 macrophages could suppress antitumor immunity and promote cancer progression ${ }^{34,35}$. $\mathrm{T}$ cells are also negatively controlled by immune checkpoint proteins, classes of molecules and signals that restrain T-cell proliferation, survival, and activation ${ }^{36}$. Although cancer cells can express tumor-specific neoantigens, thus being susceptible to be targeted by the immune system, cancer cells often express on their surface immune checkpoint molecules that suppress activation of $\mathrm{T}$ cells that could grant tumor immune surveillance. These immune checkpoint molecules, like Programmed cell-Death-1 Ligand (PD-L1) and B7, are normally found on the antigen presenting cell (APC) to avoid auto-immune $\mathrm{T}$-cell activation in the body ( $\mathrm{T}$ cells are expressing the relative receptors PD-1 and CTLA-4). Based on these observations, immunotherapies targeting these molecules have been developed and are emerging as a major breakthrough in cancer treatment ${ }^{36,37}$.

CTLA-4 is expressed on the cell membrane of $\mathrm{T}$ cells and competes with the TCR-costimulatory protein CD28 for binding to the $\mathrm{B} 7$ protein of the APC. Upregulation of CTLA-4 expression and increased CTLA-4:B7 binding results in a negative signal, which limits proliferation and survival of the $\mathrm{T}$ cells ${ }^{38}$. The exact mechanism by which anti-CTLA-4 antibodies induce an antitumor response is still imprecisely known, although preclinical evidence suggests that CTLA-4 blockade supports the activation and proliferation of a higher number of effector $\mathrm{T}$ cells and reduces TReg cell-mediated suppression of effector $\mathrm{T}$-cell response ${ }^{39,40}$. Indeed, after successful clinical trials, the anti-CTLA-4 monoclonal antibody ipilimumab was first approved for the treatment of advanced or unresectable melanoma ${ }^{41,42}$.

PD-1, a cell surface receptor, is expressed on regulatory and cytotoxic activated $\mathrm{T}$ cells in peripheral tissues while PD-L1 is mainly expressed on APC. Physiologically, 
binding of PD-L1 to its receptor results in T-cell inactivation. Thus, PD-1/PD-L1 is an immune checkpoint that guards against autoimmunity, promoting self-tolerance, and is crucial to limit immune responses in case of infections ${ }^{43,44}$. PD-1 is highly expressed on many tumorinfiltrating lymphocytes and cancer cells often overexpress PD-L1, thus escaping immune surveillance ${ }^{45}$. This has provided a strong rationale for the development of drugs targeting the PD-1/PD-L1 checkpoint. Indeed, antibodies blocking the binding of PD-L1 to its receptor, such as nivolumab and pembrolizumab, enhance immunity against a wide variety of cancers ${ }^{37,46}$. FDA rapidly approved these drugs for the treatment of melanoma, urothelial cancer, renal cell carcinoma, non-small-cell lung cancer (NSCLC), Hodgkin lymphoma, and squamous cell carcinoma of head and neck.

Unfortunately, not all cancers respond to Immune Checkpoint Inhibitor (ICI)-based therapy ${ }^{47}$. This appears to correlate very strongly with the relatively poor infiltrate of immune and inflammatory cells of ICI-resistant cancers that are, therefore, called "cold tumors" ${ }^{38}$.

\section{Combination of immune checkpoints targeting immunotherapies with DNA-damaging treatments}

A possible strategy to improve ICI-based therapy in cancer patients is to combine it with radiation or traditional, DNA-damaging, antiblastic therapies. Indeed, by induction of necrosis or immunogenic cell death (ICD), DNA damage may render cold tumors inflamed ${ }^{49}$. It was soon hypothesized that the combination of DNAdamaging radiations or drugs with ICIs could be highly beneficial to cancer patients. Increasing evidence suggests that the antitumor activity of DNA-damaging treatments is mediated not only through cytotoxic effects, but also because they stimulate immune surveillance by affecting both cancer and immune cells ${ }^{49}$.

For example, some DNA-alkylating agents, like cyclophosphamide and carboplatin, or antimetabolites, like pemetrexed, both increase the expression of MHC class I molecules on cancer cells and subvert the immunosuppressive functions of TReg cells ${ }^{50,51}$. The autocrine and paracrine circuits controlling cancer immune surveillance mainly depend on type I IFNs secreted by tumor cells and/or by tumor-infiltrating immune cells ${ }^{52}$. Recently, it has been demonstrated that DNA damaged cancer cells are an important source of type I IFNs. Cells with doublestranded DNA breaks that progress through mitosis accumulate micronuclei ${ }^{53,54}$. Micronuclear DNA is sensed by cGAS, an enzyme that catalyzes the formation of cyclic GMP-AMP (cGAMP) from ATP and GTP ${ }^{53,54}$. cGAMP, upon binding to the adaptor protein Stimulator of Interferon Genes (STING), activates the transcription factor IRF3, leading to the transcription of Type I interferons (IFNs) and, in turn, to an innate immunity response ${ }^{53-55}$.
Remarkably, using a well-described B16 syngenic mouse model of melanoma, it has been shown that irradiation of one tumor along with immune checkpoint blockade results in a T-cell-dependent growth delay of a contralateral unirradiated tumor ${ }^{53}$. The irradiated tumor produces cGAS/STING-dependent immunomodulatory signals that result in an efficient immune-mediated regression of the contralateral tumor provided that PD1/PD-L1- or CTLA-4-mediated signaling are inhibited. The results obtained in the melanoma mouse model, therefore, suggest that the immune checkpoint manipulation could indeed enhance the response to DNAdamaging, radio- and chemotherapies. Indeed, upon successful conclusion of a phase II clinical trial, one such combination treatment (pembrolizumab plus carboplatin/ pemetrexed), has been already approved for NSCLC patients $^{56}$. In any case, limitations of combining DNAdamaging radiations or drugs with ICIs appear essentially due to lack of ICI effects because of damage to immune cells by the DNA-damaging agents or, conversely, to adverse, toxic, effects of hyperactivation of inflammatory, and immunological reaction towards normal tissues ${ }^{57,58}$.

\section{Exploiting immune-dependent effects of MTAs to improve cancer treatment}

The fact that most of the spindle-targeting drugs are not so efficacious in cancer treatment has reinforced the idea that MTAs also act independently of their ability to delay mitosis completion ${ }^{13}$. How MTAs might kill cells besides their ability to induce mitotic cell death, is, however, mechanistically unclear and object of great debate ${ }^{8,13,14,59-61}$. Recent observations have indicated that when cells slip through mitosis, after mitotic delay, and adapt to paclitaxel, they form micronuclei that, as in the case of DNA-damaging treatments, activate the cGAS/ STING pathway and stimulate a proinflammatory response (Fig. 1, high taxanes) $)^{8,14,62}$. Thus, as recently suggested, taxane-based therapy may also work because it elicits the antitumor intervention of the immune system on cells escaping mitotic death ${ }^{8,63}$. Indeed, it has been shown that paclitaxel stimulates breast cancer cells to produce IFN- $\beta$ and taxane-based therapy often induces increased tumor-infiltrating immune cells, despite their suppressive effect on the rapidly cycling bone marrow cells $^{3,5,64,65}$. Thus, taxane-based therapies could benefit by the combination with ICIs. Several clinical trials have also been designed now to explore the effect of a combinatorial therapy with taxanes and ICIs. The majority of these clinical trials are still ongoing and their preliminary but very promising results are still to be definitively proven. It is a fact, however, that upon successful completion of two such trials, pembrolizumab and atezolizumab, another anti-PD-L1 monoclonal antibody, have been approved in combination with paclitaxel or its albumin-stabilized 


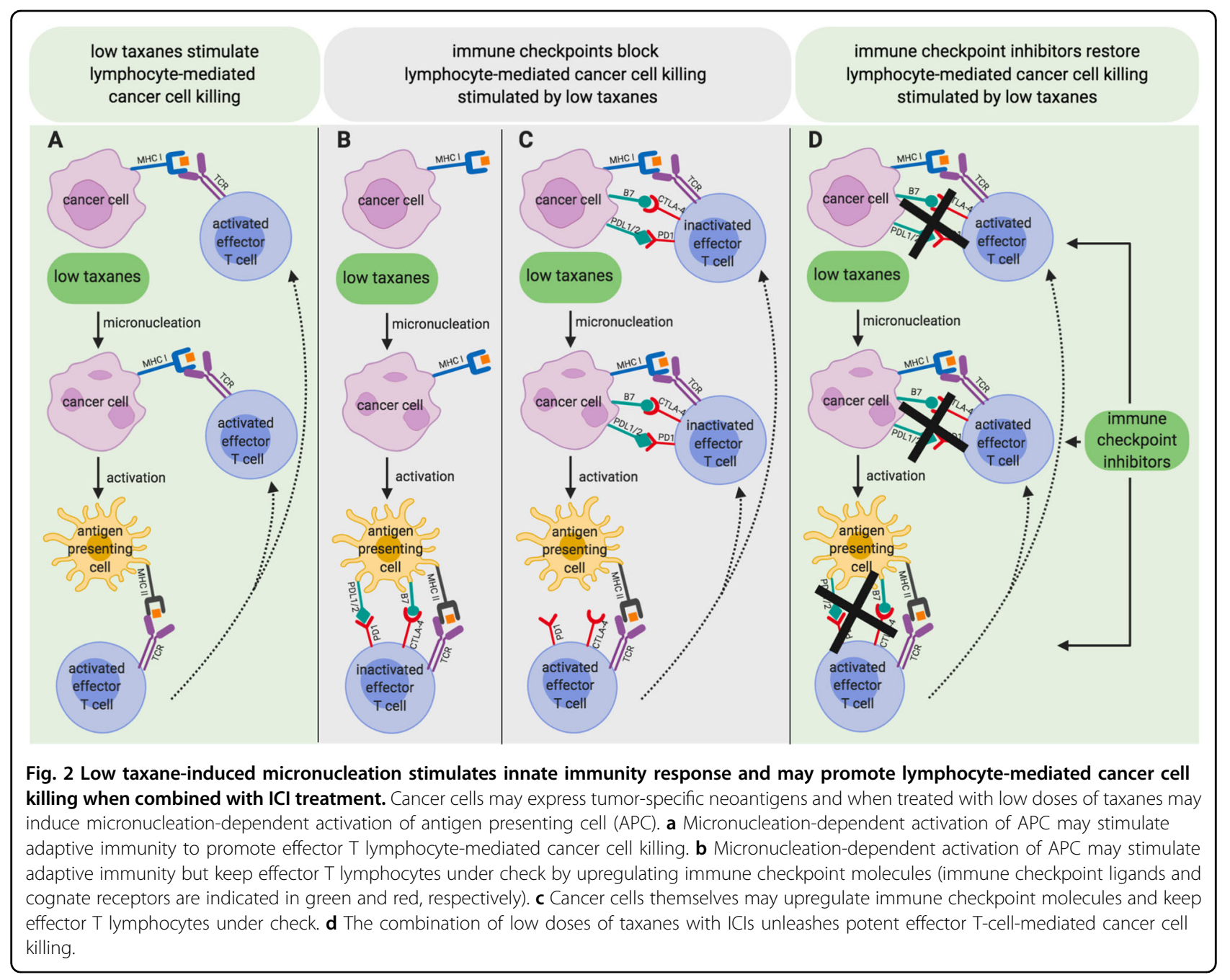

nanoparticle formulation nab-paclitaxel for the first-line treatment of metastatic squamous NSCLC ${ }^{66,67}$. Moreover, atezolizumab in combination with the sole nab-paclitaxel has also been approved for the treatment of women with unresectable triple-negative breast cancer ${ }^{68}$. The efficacy of the combination of taxanes and ICIs in cancer therapy may be explained by a simple additive effect of the two classes of drugs. However, as already discussed, the complex and not yet completely investigated immunomodulatory activity of MTAs on tumor-infiltrating immune cells might at least in part explain the success of the MTAs and ICIs combination ${ }^{63}$.

\section{Combination of ICls with low doses of MTAs}

Death after prolonged mitosis or following slippage is certainly a way MTAs kill cancer cells. However, in the case of paclitaxel, recent correlations between clinical therapeutic success for breast cancer patients and the type of mitotic aberrations induced by this drug in their breast cancer cells have indicated that the therapeutic benefit correlates with alterations in chromosome segregation rather than with prolongation of the duration of mitosis ${ }^{69}$. Indeed, while at relatively high doses paclitaxel induces mitotic delay, at much lower concentrations it does not significantly delay mitosis duration but perturbs its normal execution inducing a significant degree of chromosome missegregation and formation of micronuclei in daughter cells (Fig. 1; low taxanes) ${ }^{70}$. When single or small groups of chromosomes do not segregate with the mass of other chromosomes, they become wrapped up in nuclear membranes and remain separate from the primary nucleus ${ }^{8}$. Micronuclei formed upon chromosome segregation errors bear extensive membrane defects because 'non-core' nuclear envelope proteins, including nuclear pore complexes, do not assembly properly on lagging chromosomes ${ }^{71}$. Thus, micronuclei spontaneously and frequently lose nuclear envelope integrity, generating further DNA damage ${ }^{72}$. This micronuclear DNA can activate the cGAS-STING pathway stimulating macrophages and innate immunity and, as discussed earlier, 
innate immunity may help adaptive immunity and favor antitumor immune surveillance (Fig. 2a) ${ }^{8}$.

These observations suggest not only that a major reason for the therapeutic success of taxanes, and perhaps of other classes of MTAs, relies on their ability to promote antitumor immune surveillance but also that low doses of the drugs may be sufficient to achieve this goal. By induction of micronucleation and cGAS/STING-signaling, low doses of taxanes might be sufficient to activate innate immunity and inflammation, giving less negative side effects than standard therapeutic regimens as neutropenia and lymphopenia that may oppose to antitumor immune surveillance ${ }^{25}$. Thus, low doses of taxanes would be sufficient to enhance recruitment of immune cells and render "hot" otherwise "cold" tumors now readily attackable by the immune system (Fig. 2a). However, immune checkpoint may oppose to antitumor immune surveillance stimulated by taxanes (Fig. 2b, c). Based on these considerations, we would like to propose the use of low doses of taxanes in combination with ICIs as a strategy of wide applicability, high tolerability, and efficacy in cancer treatment (Fig. 2d).

\section{Acknowledgements}

D.G. acknowledges AIRC, Associazione Italiana per la Ricerca sul Cancro (IG grant 2017; Id. 19851 to D.G.), for support.

\section{Author details}

${ }^{1}$ CEINGE Biotecnologie Avanzate, Naples, Italy. ${ }^{2}$ DMMBM, University of Naples "Federico II", Naples, Italy. ${ }^{3}$ IEOS, CNR, Naples, Italy. ${ }^{4}$ Department of Pharmacy, University of Naples "Federico II", Naples, Italy

\section{Conflict of interest}

The authors declare that they have no conflict of interest.

\section{Publisher's note}

Springer Nature remains neutral with regard to jurisdictional claims in published maps and institutional affiliations.

Received: 26 February 2020 Revised: 16 April 2020 Accepted: 24 April 2020 Published online: 12 May 2020

\section{References}

1. Steinmetz, M. O. \& Prota, A. E. Microtubule-targeting agents: strategies to hijack the cytoskeleton. Trends Cell Biol. 28, 776-792 (2018).

2. Cao, Y. N. et al. Recent advances in microtubule-stabilizing agents. Eur. J. Med. Chem. 143, 806-828 (2018).

3. Bates, D. \& Eastman, A. Microtubule destabilising agents: far more than just antimitotic anticancer drugs. Br. J. Clin. Pharmacol. 83, 255-268 (2017).

4. Visconti, R. \& Grieco, D. Fighting tubulin-targeting anticancer drug toxicity and resistance. Endocr. Relat. Cancer 24, T107-T117 (2017).

5. Kaul, R., Risinger, A. L. \& Mooberry, S. L. Microtubule-targeting drugs: more than antimitotics. J. Nat. Prod. 82, 680-685 (2019).

6. Poruchynsky, M. S. et al. Microtubule-targeting agents augment the toxicity of DNA-damaging agents by disrupting intracellular trafficking of DNA repair proteins. Proc. Natl Acad. Sci. USA 112, 1571-1576 (2015).

7. Ogden, A., Rida, P. C., Reid, M. D. \& Aneja, R. Interphase microtubules: chief casualties in the war on cancer? Drug Discov. Today 19, 824-829 (2014).

8. Mitchison, T. J., Pineda, J., Shi, J. \& Florian, S. Is inflammatory micronucleation the key to a successful anti-mitotic cancer drug? Open Biol. 7, 170182 (2017).
9. Hayward, D., Alfonso-Pérez, T. \& Gruneberg, U. Orchestration of the spindle assembly checkpoint by CDK1-cyclin B1. FEBS Lett. 593, 2889-2907 (2019).

10. Ruan, W., Lim, H. H. \& Surana, U. Mapping mitotic death: functional integration of mitochondria, spindle assembly checkpoint and apoptosis. Front. Cell Dev. Biol. 6, 177 (2019).

11. Vitale, I., Galluzzi, L., Castedo, M. \& Kroemer, G. Mitotic catastrophe: a mechanism for avoiding genomic instability. Nat. Rev. Mol. Cell Biol. 12, 385-392 (2011).

12. Topham, C. H. \& Taylor, S. S. Mitosis and apoptosis: how is the balance set? Curr. Opin. Cell Biol. 25, 780-785 (2013).

13. Haschka, M., Karbon, G., Fava, L. L. \& Villunger, A. Perturbing mitosis for anticancer therapy: is cell death the only answer? EMBO Rep. 3, 45440 (2018).

14. Shi, J. \& Mitchison, T. J. Cell death response to anti-mitotic drug treatment in cell culture, mouse tumor model and the clinic. Endocr. Relat. Cancer 24, T83-T96 (2017).

15. Zhang, Y., Yang, S. H. \& Guo, X. L. New insights into Vinca alkaloids resistance mechanism and circumvention in lung cancer. Biomed. Pharmacother. 96, 659-666 (2017).

16. Yang, C. H. \& Horwitz, S. B. Taxol: the first microtubule stabilizing agent. Int. J. Mol. Sci. 18, 1733 (2017).

17. Visconti, R., Della Monica, R. \& Grieco, D. Cell cycle checkpoint in cancer: a therapeutically targetable double-edged sword. J. Exp. Clin. Cancer Res. 35, 153 (2016).

18. Visconti, R. et al. The FCP1-WEE1-CDK1 axis affects spindle assembly checkpoint robustness and sensitivity to antimicrotubule cancer drugs. Cell Death Differ. 22, 1551-1556 (2015).

19. Serpico, A. F. \& Grieco, D. Recent advances in understanding the role of Cdk1 in the Spindle Assembly Checkpoint. F1000Res. 9, 57 (2020).

20. Zeng, X. et al. Pharmacologic inhibition of the anaphase-promoting complex induces a spindle checkpoint-dependent mitotic arrest in the absence of spindle damage. Cancer Cell 18, 382-395 (2010).

21. Sackton, K. L. et al. Synergistic blockade of mitotic exit by two chemical inhibitors of the APC/C. Nature 514, 646-649 (2014).

22. Richeson, K. V. et al. Paradoxical mitotic exit induced by a small molecule inhibitor of APC/C ${ }^{\text {Cdc20 }}$. Nat. Chem. Biol. 10, 1038 (2010).

23. Visconti, R., Palazzo, L., Della Monica, R. \& Grieco, D. FCP1-dependent dephosphorylation is required for $\mathrm{M}$-phase-promoting factor inactivation at mitosis exit. Nat. Commun. 3, 894 (2012).

24. Serpico, A. F. et al. Wee1 rather than PIk1 is inhibited by AZD1775 at therapeutically relevant concentrations. Cancers (Basel) 6, 819 (2019).

25. Ménétrier-Caux, C., Ray-Coquard, I., Blay, J. Y. \& Caux, C. Lymphopenia in cancer patients and its effects on response to immunotherapy: an opportunity for combination with cytokines? J. Immunother. Cancer 7, 85 (2019).

26. Viudez, A. et al. Nab-paclitaxel: a flattering facelift. Crit. Rev. Oncol. Hematol. 92, 166-180 (2014).

27. Hennenfent, K. L. \& Govindan, R. Novel formulations of taxanes: a review. Old wine in a new bottle? Ann. Oncol. 17, 735-749 (2006).

28. Penna, L. S., Henriques, J. A. P. \& Bonatto, D. Anti-mitotic agents: are they emerging molecules for cancer treatment? Pharmacol. Ther. 173, 67-82 (2017)

29. Song, H., Zhou, S., Wang, R. \& Li, S. Kinesin spindle protein (KSP) inhibitors in combination with chemotherapeutic agents for cancer therapy. Chem. Med. Chem. 8, 1736-1749 (2013).

30. Doménech, E. \& Malumbres, M. Mitosis-targeting therapies: a troubleshooting quide. Curr. Opin. Pharmacol. 13, 519-528 (2013).

31. Lens, S. M., Voest, E. E. \& Medema, R. H. Shared and separate functions of pololike kinases and aurora kinases in cancer. Nat. Rev. Cancer 10, 825-841 (2010).

32. Liewer, S. \& Huddleston, A. Alisertib: a review of pharmacokinetics, efficacy and toxicity in patients with hematologic malignancies and solid tumors. Expert Opin. Investig. Drugs 27, 105-112 (2018).

33. O'Connor, O. A. et al. Randomized phase III study of alisertib or investigator's choice (selected single agent) in patients with relapsed or refractory peripheral T-cell lymphoma. J. Clin. Oncol. 37, 613-623 (2019).

34. $\mathrm{Wu}, \mathrm{Y}$., Yuan, L., Lu, Q., Xu, H. \& He, X. Distinctive profiles of tumor-infiltrating immune cells and association with intensity of infiltration in colorectal cancer. Oncol. Lett. 15, 3876-3882 (2018).

35. Segovia-Mendoza, M. \& Morales-Montor, J. Immune tumor microenvironment in breast cancer and the participation of estrogens and its receptors into cancer physiopathology. Front. Immunol. 10, 348 (2019).

36. Korman, A. J., Peggs, K. S. \& Allison, J. P. Checkpoint blockade in cancer immunotherapy. Adv. Immunol. 90, 297-339 (2006). 
37. Buchbinder, E. I. \& Desai, A. CTLA-4 and PD-1 pathways: similarities, differences, and implications of their inhibition. Am. J. Clin. Oncol. 39, 98-106 (2016).

38. Krummel, M. F. \& Allison, J. P. CD28 and CTLA-4 have opposing effects on the response of T cells to stimulation. J. Exp. Med. 182, 459-465 (1995).

39. Walker, L. S. EFIS lecture: understanding the CTLA-4 checkpoint in the maintenance of immune homeostasis. Immunol. Lett. 184, 43-50 (2017).

40. Leach, D. R., Krummel, M. F. \& Allison, J. P. Enhancement of antitumor immunity by CTLA-4 blockade. Science 271, 1734-1736 (1996).

41. Wolchok, J. D. et al. Ipilimumab monotherapy in patients with pretreated advanced melanoma: a randomised, double-blind, multicentre, phase 2, doseranging study. Lancet Oncol. 11, 155-164 (2010).

42. Hodi, F. S. et al. Improved survival with ipilimumab in patients with metastatic melanoma. N. Engl. J. Med. 363, 711-723 (2010).

43. Okazaki, T., Chikuma, S., Iwai, Y., Fagarasan, S. \& Honjo, T. A rheostat for immune responses: the unique properties of PD-1 and their advantages for clinical application. Nat. Immunol. 14, 1212-1218 (2013).

44. Freeman, G. J. et al. Engagement of the PD-1 immunoinhibitory receptor by a novel B7 family member leads to negative regulation of lymphocyte activation. J. Exp. Med. 192, 1027-1034 (2000).

45. Catakovic, K., Klieser, E., Neureiter, D. \& Geisberger, R. T cell exhaustion: from pathophysiological basics to tumor immunotherapy. Cell Commun. Signal. 15, 1 (2017).

46. Prasad, V. \& Kaestner, V. Nivolumab and pembrolizumab: monoclonal antibodies against programmed cell death-1 (PD-1) that are interchangeable. Semin. Oncol. 44, 132-135 (2017).

47. Syn, N. L., Teng, M. W. L., Mok, T. S. K. \& Soo, R. A. De-novo and acquired resistance to immune checkpoint targeting. Lancet Oncol. 18, 731-741 (2017).

48. Galon, J. \& Bruni, D. Approaches to treat immune hot, altered and cold tumours with combination immunotherapies. Nat. Rev. Drug Discov. 18, 197-218 (2019).

49. Zitvogel, L., Galluzzi, L., Smyth, M. J. \& Kroemer, G. Mechanism of action of conventional and targeted anticancer therapies: reinstating immunosurveillance. Immunity 39, 74-88 (2013).

50. Liu, W. M., Fowler, D. W., Smith, P. \& Dalgleish, A. Pre-treatment with chemotherapy can enhance the antigenicity and immunogenicity of tumours by promoting adaptive immune responses. Br. J. Cancer 102, 115-123 (2010).

51. Ghiringhelli, F. et al. Metronomic cyclophosphamide regimen selectively depletes CD4+CD25+ regulatory $T$ cells and restores $T$ and NK effector functions in end stage cancer patients. Cancer Immunol. Immunother. 56, 641-648 (2007).

52. Zitvogel, L., Galluzzi, L., Kepp, O., Smyth, M. J. \& Kroemer, G. Type I interferons in anticancer immunity. Nat. Rev. Immunol. 15, 405-414 (2015).

53. Mackenzie, K. J. et al. cGAS surveillance of micronuclei links genome instability to innate immunity. Nature 548, 461-465 (2017).

54. Harding, S. M. et al. Mitotic progression following DNA damage enables pattern recognition within micronuclei. Nature 548, 466-470 (2017).
55. Sun, L. et al. Cyclic GMP-AMP synthase is a cytosolic DNA sensor that activates the type I interferon pathway. Science 339, 786-791 (2013).

56. Langer, C. J. et al. Carboplatin and pemetrexed with or without pembrolizumab for advanced, non-squamous non-small-cell lung cancer: a randomised, phase 2 cohort of the open-label KEYNOTE-021 study. Lancet Oncol. 17, 1497-1508 (2016).

57. Fujii, T. et al. Incidence of immune-related adverse events and its association with treatment outcomes: the MD Anderson cancer center experience. Invest. N. Drugs 36, 638-646 (2018).

58. Martins, F. et al. Adverse effects of immune-checkpoint inhibitors: epidemiology, management and surveillance. Nat. Rev. Clin. Oncol. 16, 563-580 (2019).

59. Komlodi-Pasztor, E., Sackett, D. L. \& Fojo, A. T. Inhibitors targeting mitosis: tales of how drugs against a promising target were brought down by a flawed rationale. Clin. Cancer Res. 18, 51-63 (2012).

60. Weaver, B. A. How Taxol/paclitaxel kills cancer cells. Mol. Biol. Cell 25, 2677-2681 (2014)

61. Komlodi-Pasztor, E., Sackett, D., Wilkerson, J. \& Fojo, T. Mitosis is not a key target of microtubule agents in patient tumors. Nat. Rev. Clin. Oncol. 8, 244-250 (2011).

62. Kalsbeek, D. \& Golsteyn, R. M. G2/M-phase checkpoint adaptation and micronuclei formation as mechanisms that contribute to genomic instability in human cells. Int. J. Mol. Sci. 18, 2344 (2017).

63. Fong, A., Durkin, A. \& Lee, $H$. The potential of combining tubulintargeting anticancer therapeutics and immune therapy. Int. J. Mol. Sci. 20, 3 (2019).

64. Wan, S. et al. Chemotherapeutics and radiation stimulate MHC class I expression through elevated interferon-beta signaling in breast cancer cells. PLOS ONE 7, 32542 (2012).

65. Levine, M. S. \& Holland, A. J. The impact of mitotic errors on cell proliferation and tumorigenesis. Genes Dev. 32, 620-638 (2018).

66. Paz-Ares, L. et al. Pembrolizumab plus chemotherapy for squamous nonsmall-cell lung cancer. N. Engl. J. Med. 379, 2040-2051 (2018).

67. Socinski, M. A. et al. Atezolizumab for first-line treatment of metastatic nonsquamous NSCLC. N. Engl. J. Med. 378, 2288-2301 (2018).

68. Schmid, P. et al. Atezolizumab and nab-paclitaxel in advanced triple-negative breast cancer. N. Engl. J. Med. 379, 2108-2121 (2018)

69. Zasadil, L. M. et al. Cytotoxicity of paclitaxel in breast cancer is due to chromosome missegregation on multipolar spindles. Sci. Transl. Med. 6, 229-243 (2014).

70. Pineda, J. J. et al. Site occupancy calibration of taxane pharmacology in live cells and tissues. Proc. Natl Acad. Sci. USA 115, 11406-11414 (2018).

71. Liu, S. et al. Nuclear envelope assembly defects link mitotic errors to chromothripsis. Nature 561, 551-555 (2018).

72. Crasta, $\mathrm{K}$. et al. DNA breaks and chromosome pulverization from errors in mitosis. Nature 482, 53-58 (2012). 